\title{
Estructura factorial y consistencia interna de los ítems de expresión de ira del STAXI-2 en una muestra de población general de la ciudad de Monterrey, México
}

\section{Factor structure and internal consistency of the STAXI-2 anger expression items in a general population sample from Monterrey city, Mexico}

José Moral de la Rubia

Universidad Autónoma de Nuevo León
Sandra Ramos Basurto

Universidad Autónoma de Zacatecas

\section{Resumen}

Existe controversia sobre la estructura factorial de los ítems de expresión de ira del Inventario Revisado de Expresión de Ira Estado-Rasgo (STAXI-2), por consiguiente, en el presente estudio y se han propuesto tres ítems nuevos para sustituir los ítems 3, 10 y 17 con problemas de consistencia interna. Los objetivos de la investigación consistieron en contrastar el ajuste e invarianza entre ambos sexos del modelo de cuatro factores para los 24 ítems de expresión de ira del STAXI-2; en caso de problemas de consistencia interna y definición factorial, se deben explorar y contrastar modelos factoriales alternativos. La escala de expresión de ira con la inclusión de tres ítems nuevos se aplicó a una muestra no probabilística de 120 mujeres y 120 hombres de población general. Los ítems 3 y 17 mostraron problemas de consistencia interna. La correlación entre los factores de interiorización y exteriorización fue muy alta en el análisis factorial confirmatorio. El análisis paralelo de Horn y el criterio de Velicer indicaron que el número de factores es tres. Se configuraron los dos factores esperados de control y un factor no esperado con los ítems de interiorización y exteriorización (manifestación de la ira). La consistencia interna de estos tres factores varió de aceptable a alta. El ajuste a los datos del modelo de tres factores correlacionados y sus propiedades de invarianza fueron aceptables desde los índices chi cuadrada/gl, RMSEA, SRMR y CFI. Se concluye que la escala debe ser reducida a 22 ítems, ya que no trae ventajas la introducción de los ítems nuevos y su estructura es de tres factores.

Palabras clave: ira, psicometría, género, población general, México.

Nota del autor

José Moral de la Rubia, Facultad de Psicología, Universidad Autónoma de Nuevo León (UANL); Sandra Ramos Basurto, Unidad Académica de Psicología, Universidad Autónoma de Zacatecas (UAZ).

La correspondencia en relación con este artículo debe dirigirse a José Moral de la Rubia, Facultad de Psicología, UANL, calle Dr. Carlos Canseco, número 110, colonia Mitras, C. P. 64460, Monterrey, Nuevo León, México.

Dirección electrónica: jose_moral@hotmail.com 


\begin{abstract}
There exits controversy about the factor structure of the anger expression items from the Revised StateTrait Anger Expression Inventory (STAXI-2). Three new items have been proposed to replace items 3, 10 and 17 with problems of internal consistency. The aims of this study were to contrast the fit and invariance of the four-factor model for the 24 anger expression items from the STAXI-2 across sex; in case of problems of internal consistency and factor definition, explore and contrast alternative factor models. The STAXI-2 anger expression scale with the inclusion of three new items was administrated to a non-probability sample of 120 women and 120 men from the general population. Items 3 and 17 showed internal consistency problems. The correlation between internalizing and externalizing factors was very high in the confirmatory factor analysis. Horn's parallel analysis and Velicer's criterion indicated that the number of factors is three. The expected two factors of control, and a non-expected factor with the internalizing and externalizing items (anger expression) were configured. The internal consistency of these three factors varied from acceptable to high. The fit to the data of 3-factor model and its properties of invariance across sex were acceptable by the chi-square/gl, RMSEA, CFI and $S R M R$ indexes. It is concluded that the scale should be reduced to 22 items, does not bring advantages the introduction of the new items, and its structure is three factors.
\end{abstract}

Keywords: Anger, psychometrics, gender, general population, Mexico.

Dentro de la dimensión del afecto negativo se ubica la ira. La ira es una emoción activadora que surge como una reacción a la vulnerabilidad ante una amenaza, coerción o daño recibido o frente a una situación de frustración o trato diferencial (Carver \& Harmon-Jones, 2009). La ira se puede expresar con una conducta de agresión dirigida contra los demás o puede ocultarse cara a los demás e incluso dirigirse contra sí mismo. También cabe distinguir entre el control de la intensidad de la ira expresada contra los demás y el control de la intensidad de la misma guardada ante los demás y dirigida contra sí mismo (Welty, 2011).

Entre los instrumentos para su evaluación, destaca el Inventario de Expresión de Ira Estado-Rasgo (STAXI). En su forma revisada de 49 ítems (STAXI-2), el inventario incluye una escala de estado de ira con tres factores (emocional, verbal y física); una escala de rasgo de ira con dos factores (temperamento y reacción); y una escala de expresión de ira con cuatro factores (interiorización o ira contenida, exteriorización o ira dirigida contra los demás, control de la ira ocultada ante los demás y control de la ira dirigida contra los demás) (Spielberger, 1999). El STAXI-2 ha sido validado en varios países como México (Oliva, Hernández \& Calleja, 2010), España (Miguel-Tobal, Casado, Cano, \& Spielberger, 2006), Francia (Borteyrou, Bruchon-Schweitzer, \& Spielberger, 2008) y Alemania (Etzler, Rohrmann, \& Brandt, 2014), entre otros.

Con la versión latinoamericana del STAXI-2, aplicada a una muestra incidental de psicólogos de varios países hispanoparlantes, Moscoso 
(2000) observó que los 24 ítems de expresión de la ira se dividieron en tres factores en hombres (control de la ira, expresión de la ira manifiesta y expresión de la ira contenida) y en cuatro conforme a la expectativa en mujeres. La consistencia interna varió de .61 a .99. Con esta misma versión del STAXI-2, Alcázar, Deffenbacher y Byrne (2011), en una muestra de 503 estudiantes universitarios mexicanos, obtuvieron cinco factores con los 24 ítems de expresión de ira. Los seis ítems de expresión contenida o interiorización se dividieron en dos factores (evitación de las otras personas/ sentimientos contenidos y rumiación) y se reprodujeron los otros tres factores en su interpretación (control de la ira manifiesta, control de la ira contenida y expresión de la ira manifiesta), aunque con variaciones en su configuración. Los valores de consistencia interna variaron de .70 a .83 , salvo aquellos de los dos factores de interiorización, el de evitación tuvo una consistencia interna baja ( $\alpha$ $=.61) \mathrm{y}$ el de sentimientos contenidos muy baja $(\alpha=.50)$.

Con traducción al español del STAXI-2, realizada por Miguel-Tobal et al. (2006), Oliva et al. (2010), en una muestra incidental de 865 estudiantes mexicanos, obtuvieron los cuatro esperados con los 24 ítems de expresión de ira. La consistencia interna fue alta en los dos de control y baja en los dos de expresión. El ajuste a los datos del modelo de cuatro factores correlacionados fue aceptable: $\chi^{2}(276, N=$
$865)=507.28, p<.001, \chi^{2} / g l=2.20, C F I=$ .95 y $R M S E A=.05$. Estos resultados fueron convergentes con los estudios de Miguel-Tobal et al. (2006) y Del Barrio, Aluja y Spielberger (2004) en España.

Asimismo, en traducción al español del STAXI-2, realizada por el mismo Miguel-Tobal et al. (2006), además de Moral, González y Landero (2010), en una muestra de 226 amas de casa mexicanas, observaron problemas de consistencia interna y configuración factorial en tres de los 24 ítems de expresión de ira (ítems 3 , 10 y 17) y problemas para reproducir el número de factores y la configuración conforme a las expectativas. Al eliminar estos tres ítems, se incrementó la consistencia interna de la escala y su factor (interiorización o expresión de la ira ocultada a los demás), el número de factores por el criterio de Kaiser fue cuatro, como se esperaba, y se configuraron los factores esperados tras una rotación oblicua en el análisis factorial exploratorio. La consistencia interna de los factores de control fue alta $(\alpha=.86$ control de la ira dirigida hacia los demás y .88 control para la ira ocultada a los demás), aceptable la del factor de exteriorización $(\alpha=.74)$ y baja la del factor de interiorización $(\alpha=.68)$. El ajuste de un modelo de cuatro factores correlacionados fue aceptable por máxima verosimilitud: $\chi^{2}(184$, $N=226)=290.97, p<.01, \chi^{2} / g l=1.58, R M S E A$ $=.05, N F I=.84, N N F I=.92$ y $C F I=.93$.

Los ítems 3, 10 y 17 se refieren a una conciencia de activación por la ira mayor de la 
que se exterioriza, incluso de lo que la persona quiere admitir frente a sí misma. Moral et al. (2010) proponían como nuevos ítems "ardo por dentro, aunque no lo muestro", "me irrito más de lo que la gente piensa" y "estoy más enfadado/a de lo que quiero admitir". También proponían probar ítems de somatización de la emoción que hicieran referencia a una conciencia de activación por ira contenida, por ejemplo, "me duele cabeza", "me siento cansado/a por el enojo que siento", "grito por dentro de rabia".

Además, con traducción al español del STAXI-2, realizada por Miguel-Tobal et al. (2006), Moral y Segovia (2015), en una muestra de 200 mujeres mexicanas con VIH, observaron problemas de consistencia interna en los ítems 3 y 17. Por el análisis paralelo de Horn y el criterio de Velicer se definieron tres factores; extraídos por Ejes Principales y rotación Promax, se obtuvieron el de control interno con seis ítems $(\alpha=.88)$ y el de control externo con seis ítems $(\alpha=.79)$, respectivamente, así como uno nuevo de expresión de la ira con 10 ítems que resultó de la unión de exteriorización e interiorización sin los ítems 3 y $17(\alpha=.89)$. El ajuste por máxima verosimilitud de este modelo de tres factores y 22 ítems fue aceptable: $\chi^{2} / g l=601.17 / 206=$ 2.92, RMSEA $=.09$, SRMR $=.08$ y $C F I=.82$.

Con el objetivo de evaluar la ira como una variable de personalidad bajo el modelo de Spielberger, el STAXI-2 podría ser reducido a la escala de expresión de la ira, con lo cual se disminuiría a la mitad el número de ítems apli- cados, como sugieren Moral et al. (2010). No obstante, con respecto a la estructura factorial de esta escala, se observan discrepancias entre los estudios realizados en México (Alcázar et al., 2011; Moral et al., 2010; Moral \& Segovia, 2015; Oliva et al., 2010). Otra limitación para la recomendación de su uso en este país es que las propiedades psicométricas de la escala no han sido estudiadas en una muestra de adultos de población general. Aunque se ha explorado la estructura factorial de los 24 ítems de expresión de la ira en ambos sexos (Moscoso, 2000), no se ha contrastado la invarianza del modelo de cuatro factores correlacionados, propuesto para la escala en México u otros países.

Al considerar dichos antecedentes, el presente artículo tiene como objetivos: 1) estimar la consistencia interna de los 24 ítems y de los cuatro factores de la escala de expresión de la ira (AX) del STAXI-2 en una muestra de adultos de población general de la ciudad de Monterrey, México; 2) contrastar el ajuste del modelo original de cuatro factores correlacionados en la muestra conjunta y su invarianza entre ambos sexos; 3) contrastar el ajuste del modelo de cuatro factores correlacionados en la muestra conjunta y su invarianza entre ambos sexos, al eliminar parámetros no significativos o ítems con problemas de consistencia interna; 4) contrastar el ajuste del modelo de cuatro factores correlacionados en la muestra conjunta y su invarianza entre ambos sexos, al sustituir los ítems de interiorización con baja 
consistencia interna por los ítems sugeridos por Moral et al. (2010); 5) si el ajuste del modelo original de cuatro factores correlacionados es malo y presenta variación significativa entre ambos sexos, aun eliminando parámetros no significativos o sustituyéndolos por los ítems sugeridos por Moral et al. (2010); explorar modelos factoriales alternativos, con base en la exploración con un número de ítems menor que los 24 ítems originales tras eliminar aquellos con problemas de consistencia interna y configuración factorial, o con un número equivalente una vez sustituidos los ítems de interiorización con baja consistencia interna por los ítems sugeridos por Moral et al. (2010); y 6) especificar modelos factoriales alternativos derivados del análisis factorial exploratorio, además de contrastar su ajuste en la muestra conjunta e invarianza entre ambos sexos.

Se espera una estructura de cuatro factores correlacionados, con un ajuste adecuado en la muestra conjunta (Oliva et al., 2010) o de tres factores (Moscoso, 2000; Moral \& Segovia, 2015); unos valores de consistencia interna altos para los 24 ítems y los dos factores de control $(\alpha \geq .80)$; y unos valores de consistencia interna aceptables para los dos factores de expresión de .79 a .70 (Moscoso \& Spielberger, 1999; Oliva et al., 2010). Aunque la consistencia interna del factor de expresión de ira ocultada a los demás o interiorización podría ser baja, de .69 a .60 , o incluso muy baja, de .59 a .50 (Alcázar et al., 2011), por efecto de los ítems 3, 10 y 17
(Moral et al., 2010; Moral \& Segovia, 2015). La consistencia interna del factor de interiorización podría incrementarse con la sustitución de estos ítems, si se usan los ítems sugeridos por Moral et al. (2010), asimismo, con esta sustitución podría mejorar la configuración, ajuste e invarianza entre ambos sexos del modelo esperado de cuatro factores correlacionados.

\section{Método}

\section{Participantes}

Los criterios de inclusión fueron los siguientes: ser mayor de edad, residir en la ciudad de Monterrey, Nuevo León, México, saber leer y escribir, así como dar su consentimiento informado. Los criterios de exclusión, en cambio fueron: cuestionario incompleto $\mathrm{o}$ actitud inatenta o arbitraria de los participantes, según la percepción de la encuestadora.

El procedimiento de muestreo fue no probabilístico, con base en una muestra por cuotas de sexo de 240 participantes voluntarios; ésta quedó integrada por 120 mujeres y 120 hombres. Durante la aplicación se alternó entre un hombre y una mujer para conseguir el mismo número de participantes de ambos sexos. El porcentaje de participantes que dio el consentimiento y respondió al cuestionario fue del 82\% (240 de 293). En la mayoría de los casos donde no se consintió o no se deseó participar, la falta de tiempo fue la causa principal. 
$\mathrm{El}$ inventario fue administrado de forma individual en casas, calles peatonales y parques públicos. Los participantes contestaron de forma manuscrita sentados en bancas (calles y parques) o en el interior de sus casas (salas o comedores). La aplicación fue realizada por una psicóloga, quién permanecía cerca del participante mientras el inventario era contestado por si surgían dudas o preguntas. Al recoger el inventario, se revisó que estuviese totalmente respondido. Si había preguntas sin responder, se pedía amablemente que se respondiesen. La colecta de datos se realizó entre enero y octubre de 2013 en la ciudad de Monterrey, México.

La media de edad fue 35.41 años $(D E=$ 9.63), con mínima de 18 años y máxima de 57 años. Respecto a la escolaridad, 23 de los 240 participantes $(9.6 \%)$, indicaron haber cursado primaria; 58 (24.2\%) secundaria; 47 (19.6\%) media superior general; 39 (16\%) media superior técnica; y 73 (30.4\%) estudios superiores. Con relación a la actividad laboral, 45 de los 240 participantes (18.8\%), reportaron dedicarse a labores de su hogar; 67 (27.9\%) dijeron trabajar como empleados de oficina o ventas; 54 (22.5\%), trabajar como empleados manuales; 45 (18.8\%), desempeñarse como empleados profesionistas; $14(5.8 \%)$, tener negocio propio; 8 (3.3\%) estar desempleados; y 7 (2.9\%), dedicarse a estudiar. Acerca del estatus socioeconómico autodefinido, 54 de los 118 participantes (22.5\%) se definieron de estatus socioeconómico bajo; 62 (25.8\%), medio-bajo; 91 (37.9\%), medio- medio; 30 (12.5\%), medio-alto; y 3 (1.3\%) alto. En tanto, en lo referente al estado civil, 118 de los 240 participantes (49.2\%) reportaron estar casados; 22 (9.2\%), solteros; 44 (18.3\%), divorciados o separados; y 56 (23.3\%), en unión libre. Con respecto al lugar de residencia, 121 de los 240 participantes (50.4\%) afirmaron vivir en familia con hogar propio; 46 (18.8\%), con la familia de origen propia; 44 (18.7\%), solos; 23 (9.6\%), como familia monoparental; y $6(2.5 \%)$, con la familia de origen de su pareja. De los 240 participantes, 191 (79.6\%) dijeron tener hijos, no así el resto, 49 (20.4\%). La media del número de hijos fue $2.31(D E=1.06)$, con variaciones de uno a cinco entre quienes sí tenían.

\section{Instrumentos}

Se utilizó la escala de Expresión de Ira (AX) del Inventario de Expresión de Ira EstadoRasgo, en su segunda edición (STAXI-2-AX) de Spielberger (1999), con la traducción al español de Miguel-Tobal et al. (2006). Configurada por 24 ítems tipo Likert con un rango de uno (en absoluto) a cuatro (muchísimo). Proporciona un índice general sobre la frecuencia con la cual se manifiesta ira (IRA). Esta escala se divide en cuatro subescalas de seis ítems cada una: Control Externo o de la exteriorización de ira (CEX), que evalúa la frecuencia del control de ira dirigida contra los demás (ítems $1,5,8,11$, 16 y 18); Control Interno o de la ira contenida (CIN), cuya función es evaluar maniobras emprendidas por la persona para controlar una ira guardada ante los demás (ítems 19, 20, 21, 
22, 23 y 24); Exteriorización (EXT), determina la frecuencia con que la persona manifiesta la ira contra otras personas (ítems 2, 4, 6, 9, 13, 15); e Interiorización (INT), estima la frecuencia con que los sentimientos de ira se esconden ante los demás de forma consciente (ítems 3, $7,10,12,14$ y 17). Además se incluyeron tres ítems adicionales: ítem 25: "me duele cabeza por la rabia que siento", ítem 26: "me siento cansado/a por el enojo que siento" e ítem 27: "grito por dentro de rabia", dirigidos a evaluar interiorización o expresión de ira contenida (INT). Véase apéndice.

\section{Procedimiento}

La participación fue voluntaria y no remunerada. Al momento de solicitar el consentimiento para la participación en el estudio por medio de una carta escrita, se informaba de los objetivos de la investigación, quiénes eran los responsables de la misma y su adscripción institucional, además se garantizaba el anonimato y confidencialidad de la información dada, según las normas éticas de la Sociedad Mexicana de Psicología (2007). En caso de que la persona deseara participar, señalaba la casilla correspondiente sus motivos; y en caso contrario, aparte de señalar la casilla indicada para eso, se le pedía expresar sus motivos por escrito.

\section{Análisis de datos}

En relación con el primer objetivo, la consistencia interna se calculó por el coeficiente alfa de Cronbach $(\alpha)$; unos valores de $\alpha \geq .90$ se consideraron excelentes o muy altos; de .80 a .89 , buenos o altos; de .70 a .79, aceptables; de .60 a .69 , cuestionables o bajos; de .50 a .59 , pobres o muy bajos; $\mathrm{y}<.50$, inaceptables (Cronbach \& Shavelson, 2004).

En relación con los objetivos dos, tres, cuatro y seis, se especificaron y contrastaron modelos factoriales. Para contrastar la invarianza de los modelos factoriales entre ambos sexos (análisis multigrupo), se definieron cuatro modelos anidados con constricciones acumulativas: sin constricciones, con constricciones en los pesos de medida, en las varianzas-covarianzas estructurales y en los residuos de medida. Los parámetros fueron estimados por Máxima Verosimilitud, tanto en el análisis unigrupo (muestra total) como en el análisis multigrupo. Se contemplaron cinco índices de ajuste para el análisis factorial confirmatorio: prueba chicuadrado de Pearson $\left(\chi^{2}\right)$, cociente entre el estadístico chi-cuadrado y sus grados de libertad $\left(\chi^{2} / g l\right)$, índice comparativo de ajuste (CFI) de Bentler y Bonett, error de aproximación cuadrático medio (RMSEA) de Steiger y Lind, así como el residuo estandarizado cuadrático medio (SRMR) de Jöreskog y Sörbom. Al incumplirse el supuesto de normalidad multivariada (valor estandarizado de la curtosis multivariada de Mardia > 2), se complementó el contraste de la significación de los parámetros por el método de percentiles corregidos de sesgo (PCS) y de la bondad de ajuste global por prueba de BollenStine ( $p$ de B-S). Se estipularon como valores de buen ajuste: $p$ de $\chi^{2}$ у $p$ de $\mathrm{B}-\mathrm{S}>.05, \chi^{2} / g l \leq 2$, $C F I \geq .90, S R M R$ y $R M S E A \leq .05$. Se acordaron 
como valores de ajuste aceptable: $p$ de $\chi^{2}$ y $p$ de $\mathrm{B}-\mathrm{S}>.01, \chi^{2} / g l \leq 3, C F I \geq .80, S R M R$ y $R M S E A$ $\leq .09$ (Byrne, 2010 .

En relación con el quinto objetivo, se usó análisis factorial exploratorio. Para determinar el número de factores, se empleó el criterio de Horn (percentil 95) y el criterio de Velicer (promedio mínimo de las correlaciones parciales al cuadrado, parcializando los componentes extraídos). Se partió de la matriz de correlaciones policóricas. La extracción de factores se hizo por Ejes Principales y la rotación de la matriz factorial por Promax.

Los cálculos se realizaron con SPSS21 y AMOS16. Para el análisis paralelo de Horn, el de Velicer y las correlaciones policóricas se empleó el menú R, versión 2 para SPSS.

\section{Resultados}

\section{Consistencia interna de los 24 ítems y cuatro factores de la escala de ira del STAXI-2}

En la muestra conjunta, la consistencia interna de los 24 ítems fue alta $(\alpha=.85)$ y se incrementó a .87 con la eliminación de los ítems 3 y 17 , al igual que en la muestra de mujeres (de .83 a .85) y de hombres (de .88 a .89). Asimismo, los seis ítems de control interno y los seis ítems de control externo de esta muestra tuvieron consistencia interna alta $(\alpha=.86$ y .80 , respectivamente), como sucedió también en la muestra de mujeres ( $\alpha=.84$ y .82, respectivamente); en hombres, por el contrario, control interno tuvo consistencia interna alta $(\alpha=.89)$ y control externo aceptable $(\alpha=.78)$.

En la muestra conjunta, la consistencia interna de los seis ítems de exteriorización fue baja $(\alpha=.63)$ y no se incrementó con la eliminación ningún ítem, al igual que en hombres $(\alpha=.64)$; a diferencia, en mujeres fue muy baja $(\alpha=.58)$, pero se incrementó a baja con la eliminación del ítem cuatro $(\alpha=.61)$.

La consistencia interna de los seis ítems de interiorización fue baja $(\alpha=.65)$ en la muestra conjunta, pero pasó a ser aceptable con la eliminación de los ítems 3 y $17(\alpha=.70)$, al igual que en hombres (de .69 a .74); en mujeres se incrementó, pero sigue siendo baja (de .61 a .67).

\section{Contraste e invarianza del modelo original de cuatro factores correlacionados}

Al contrastar el modelo de cuatro factores correlacionados con sus ítems originales (seis indicadores para cada factor) en la muestra conjunta (análisis unigrupo), la solución fue admisible. La carga de interiorización sobre el ítem 17 no fue significativa por $\operatorname{ML}(p=$ $.063)$, pero sí por $P C S(p=.027)$, asimismo, la varianza de interiorización no fue significativa por $M L(p=.166)$, pero sí por $\operatorname{PCS}(p=$ .001). La correlación entre interiorización y exteriorización fue muy alta $(r=.77, p<.001$ por $M L)$. La bondad de ajuste fue rechazada por la prueba chi-cuadrada y la de Bollen-Stine. Los demás índices mostraron valores de ajuste aceptables (ver tabla 1).

Al contrastar la invarianza del modelo factorial entre ambos sexos (análisis multigrupo), 
las soluciones fueron admisibles, empero, se observaron problemas importantes en el factor de interiorización. Por el método $M L$, en el modelo sin constricciones, la varianza de interiorización y sus correlaciones con los otros tres factores no fueron significativas en mujeres y hombres; los pesos del factor sobre los ítems 7, 10 y 17 no fueron significativos en mujeres y el factor no tuvo un peso significativo sobre ninguno de sus seis indicadores en hombres. Al constreñir los pesos estructurales, la no significancia de la varianza de interiorización y del peso de interiorización sobre el ítem 17 persistió en ambos sexos, así como la no significancia de la correlación entre interiorización y control externo en mujeres y de la correlación entre interiorización y control interno en hombres. Al constreñir las varianzas-covarianzas y los residuos, la no significancia de la varianza de interiorización persistió. Por el método de $P C S$, sólo la carga de interiorización sobre el ítem 17 no fue significativa en el modelo sin constricciones en mujeres, este parámetro pasó a ser significativo con la constricción de los pesos estructurales. En este contraste, el peso del factor de exteriorización sobre el ítem cuatro no fue significativo en mujeres por $M L$ y $P C S$ y en hombres por $P C S$, pero pasó a ser significativo con la constricción de los pesos estructurales por $M L$ y $P C S$. La correlación entre interiorización y exteriorización fue mayor que .70 en los modelos con constricciones en las varianzascovarianzas y pesos estructurales en ambos sexos, y en los modelos sin constricciones y con constricciones en los pesos estructurales en hombres. Así también, la correlación entre control interno y control externo fue mayor que .70 en los modelos $\sin$ constricciones $y$ con constricciones en los pesos estructurales en hombres. Los índices de ajuste fueron malos en el modelo sin constricciones y éstos empeoraron en los modelos con constricciones, salvo RMSEA y $\chi^{2} / g l$ (ver tabla 1$)$.

\section{Contraste e invarianza del modelo de cuatro} factores correlacionados, una vez eliminados los ítems con problemas de consistencia interna

Se volvió a contrastar el ajuste del modelo de cuatro factores correlacionados en la muestra conjunta y su invarianza entre mujeres y hombres fue contrastado nuevamente, aunque se eliminaron los ítems 3 y 17, debido a la mejora que así se reflejaba en la consistencia interna del factor de interiorización y de la escala, y a que el ítem 17 resultó no significativo en todos los modelos en ambas muestras.

En el análisis unigrupo, la solución fue admisible. Todos los parámetros fueron significativos por ML y PCS. La correlación entre interiorización y exteriorización fue muy alta $(r=.79, p<.001$ por $M L)$, pero las otras tres correlaciones fueron menores que .70. La bondad de ajuste fue rechazada por la prueba chi-cuadrada y de la Bollen-Stine. Los demás índices mostraron un ajuste aceptable (ver tabla 2). La bondad de ajuste de este modelo con 22 ítems y cuatro factores fue significativamente mejor que la del modelo original con 24 ítems y cuatro factores $\left(\Delta \chi^{2}[43, N=240]=116.89, p\right.$ $<.001)$.

En el análisis multigrupo, las soluciones fueron admisibles. Todos los parámetros 
fueron significativos por $M L$ y $P C S$, salvo el peso de exteriorización sobre el ítem cuatro en el modelo sin constricciones en mujeres. La correlación entre los factores de interiorización y exteriorización fue muy alta en todos los modelos y en ambas muestras, al variar de .70 a .85 , y de .79 una vez constreñidas las varianzascovarianzas estructurales. La bondad de ajuste no fue aceptada por la prueba chi-cuadrada y la de Bollen-Stine en todos los modelos. Los demás índices reflejaron un ajuste de aceptable en el modelo sin constricciones, mismos que empeoró significativamente en los modelos con constricciones anidadas (ver tabla 2). En comparación con el contraste de la invarianza del modelo original con 24 ítems, dicho modelo con 22 ítems y cuatro factores mostró menos problemas de significación de parámetros y mejores valores de ajuste.

Tabla 1

Índices de ajuste del modelo original de cuatro factores correlacionados

\begin{tabular}{cccccc}
\hline Índices & Uni- & \multicolumn{4}{c}{ Multigrupo (mujeres y hombres) } \\
\cline { 3 - 6 } de ajuste & grupo & SC & PM & VE & RM \\
\hline$X^{2}$ & 568.345 & 945.685 & 977.505 & 999.611 & 1039.297 \\
$g l$ & 246 & 492 & 512 & 522 & 546 \\
$p$ & $<.001$ & $<.001$ & $<.001$ & $<.001$ & $<.001$ \\
$X^{2} / g l$ & 2.310 & 1.922 & 1.909 & 1.915 & 1.903 \\
$p$ de B-S & 0 & 0 & 0 & .001 & 0 \\
CFI & .822 & .771 & .765 & .759 & .751 \\
RMSEA & .074 & .062 & .062 & .062 & .062 \\
SRMR & .078 & .094 & .098 & .104 & .105 \\
\hline
\end{tabular}

Nota: Modelos anidados con constricciones acumulativas: $\mathrm{SC}=\sin$ constricciones, $\mathrm{PM}=$ con constricciones en pesos de medida, $\mathrm{VE}=$ en varianzas-covarianzas estructurales y $\mathrm{RM}=$ en residuos de medida.

Tabla 2

Índices de ajuste del modelo de cuatro factores correlacionados (sin items 3 y 17)

\begin{tabular}{cccccc}
\hline Índices & Uni- & \multicolumn{4}{c}{ Multigrupo (mujeres y hombres) } \\
\cline { 3 - 6 } de ajuste & grupo & SC & PM & VE & RM \\
\hline$X^{2}$ & 451.456 & 760.48 & 791.14 & 814.18 & 847.76 \\
$g l$ & 203 & 406 & 424 & 434 & 456 \\
$p$ & $<.001$ & $<.001$ & $<.001$ & $<.001$ & $<.001$ \\
$X^{2} / g l$ & 2.224 & 1.87 & 1.87 & 1.88 & 1.86 \\
$p$ de B-S & 0 & .001 & .001 & .001 & .001 \\
CFI & .856 & .810 & .804 & .797 & .790 \\
RMSEA & .076 & .061 & .060 & .061 & .060 \\
SRMR & .071 & .085 & .090 & .097 & .098 \\
\hline
\end{tabular}

Nota: Modelos anidados con constricciones acumulativas: $\mathrm{SC}=$ sin constricciones, $\mathrm{PM}=$ con constricciones en pesos de medida, $\mathrm{VE}=$ en varianzas-covarianzas estructurales y $\mathrm{RM}=$ en residuos de medida. 
Contraste e invarianza del modelo de cuatro factores correlacionados, una vez sustituidos los ítems con problemas de consistencia interna

Al estimar la consistencia interna de los seis ítems originales de interiorización y los tres nuevos se obtuvo un valor aceptable entre los nueve ítems $(\alpha=.78)$. Con la eliminación de los ítems 3 y 17 se incrementó a un valor de consistencia bueno $(\alpha=.81)$. Desde los valores de correlación de cada ítem con el resto de la escala se observó que el más débil fue el ítem 10. Para que quedaran seis ítems en la escala de interiorización se excluyó el ítem 10 y entraron los tres nuevos. La consistencia interna de los seis ítems fue buena $(\alpha=.81)$.

El modelo de cuatro factores correlacionados con seis indicadores cada factor fue contrastado en la muestra conjunta (análisis unigrupo). La solución fue admisible. Todos los parámetros fueron significativos por $M L$ y PCS. La correlación entre exteriorización e interiorización fue muy alta $(r=.79, p<.001$ por $M L)$, las otras presentaban correcciones valores menores que .70. La bondad de ajuste se rechazó por la prueba chi-cuadrada y de
Bollen-Stine. Los índices mostraron un ajuste aceptable (ver tabla 3). La bondad de ajuste de este modelo de cuatro factores con 24 ítems fue significativamente peor que la del modelo de cuatro factores sin los ítems 3 y $17\left(\Delta \chi^{2}[43, N=\right.$ $240]=132.52, p<.001)$

Al contrastar la invarianza del modelo entre ambos sexos (análisis multigrupo), las soluciones fueron admisibles. Todos los parámetros fueron significativos en los cuatro modelos anidados y las dos muestras por ML y PCS, salvo el peso del factor de exteriorización sobre el ítem cuatro en el modelo sin constricciones en mujeres por ambos métodos. La correlación entre interiorización y exteriorización de la ira fue mayor que .70 en los cuatro modelos anidados en las dos muestras; en tanto, la correlación entre control interno y control externo fue mayor que .70 en los modelos sin constricciones y con constricciones en los pesos de medida en hombres. Todas las demás correlaciones fueron menores que .70. La bondad de ajuste fue mala en el modelo sin constricciones y empeoró significativamente en los modelos anidados (ver tabla 3). 


\section{Tabla 3}

Índices de ajuste del modelo de cuatro factores correlacionados con 3 items de interiorización sustituidos

\begin{tabular}{cccccc}
\hline Índices & Uni- & \multicolumn{4}{c}{ Multigrupo (mujeres y hombres) } \\
\cline { 3 - 6 } de ajuste & grupo & $\mathrm{SC}$ & $\mathrm{PM}$ & $\mathrm{VE}$ & $\mathrm{RM}$ \\
\hline $\mathrm{X}^{2}$ & 583.977 & 944.679 & 974.889 & 988.566 & 1044.305 \\
$g l$ & 246 & 492 & 512 & 522 & 546 \\
$p$ & $<.001$ & $<.001$ & $<.001$ & $<.001$ & $<.001$ \\
$\mathrm{X}^{2} / g l$ & 2.374 & 1.920 & 1.904 & 1.894 & 1.913 \\
$p$ de B-S & 0 & .001 & .001 & .001 & .001 \\
CFI & .839 & .802 & .797 & .795 & .782 \\
RMSEA & .076 & .062 & .062 & .061 & .062 \\
SRMR & .073 & .090 & .096 & .101 & .104 \\
\hline
\end{tabular}

Nota: Modelos anidados con constricciones acumulativas: $\mathrm{SC}=$ sin constricciones, $\mathrm{PM}=$ con constricciones en pesos de medida, $\mathrm{VE}=$ en varianzas-covarianzas estructurales y $\mathrm{RM}=$ en residuos de medida.

\section{Exploración de la estructura factorial}

\section{Con 22 ítems, excluidos los ítems 3 y 17.}

Por los claros problemas de consistencia interna y configuración factorial se optó por explorar la estructura de la escala sin los ítems 3 y 17. El análisis paralelo de Horn y el promedio mínimo de las correlaciones parciales al cuadrado coincidieron en mostrar tres factores sustantivos en la muestra conjunta y de mujeres. En las dos muestras se configuraron los dos factores esperados de control: interno (ítems $19,20,21,22,23$ y 24) y externo (ítems 1, 5,
$8,11,16$ y 18), y un factor de expresión de ira (ítems 2, 4, 6, 7, 9, 10, 12, 13, 14 y 15). El porcentaje de varianza explicada fue de $39.3 \%$ en la muestra conjunta y $37.6 \%$ en la muestra de mujeres. Las correlaciones entre los factores fueron significativas en las dos muestras, al variar de -.30 a .57. En hombres, ambos criterios convergieron en dos factores y se configuró un factor de control y otro de expresión de ira; la correlación entre ambos factores fue de -.46. El porcentaje de varianza explicada fue de $39.8 \%$ (ver tabla 4 ). 
Tabla 4

Matrices de configuración con cargas factoriales $\geq .30$

\begin{tabular}{|c|c|c|c|c|c|c|c|c|c|}
\hline \multirow{2}{*}{ Ítems } & \multirow{2}{*}{ Exp. } & \multicolumn{3}{|c|}{ Conjunta } & \multicolumn{3}{|c|}{ Mujeres } & \multicolumn{2}{|c|}{ Hombres } \\
\hline & & F1 & $\mathrm{F} 2$ & F3 & F1 & F2 & F3 & $\mathrm{F} 1$ & $\mathrm{~F} 2$ \\
\hline 1 & CEX & & & .466 & & .641 & & .508 & -.322 \\
\hline 2 & EXT & & .384 & & & & .351 & & .346 \\
\hline 4 & EXT & & .313 & & & & .300 & & .352 \\
\hline 5 & CEX & & & .548 & & .599 & & .329 & \\
\hline 6 & EXT & & .444 & & .387 & & .409 & & .530 \\
\hline 7 & INT & & .504 & .307 & & & .347 & & .391 \\
\hline 8 & CEX & & & .594 & & .661 & & .340 & \\
\hline 9 & EXT & & .311 & & & & .351 & & .303 \\
\hline 10 & INT & & .542 & & & & .344 & & .654 \\
\hline 11 & CEX & & & .474 & & .642 & & .311 & \\
\hline 12 & INT & & .682 & & & & .755 & & .662 \\
\hline 13 & EXT & & .575 & & & & .487 & & .715 \\
\hline 14 & INT & & .776 & & & & .668 & & .777 \\
\hline 15 & EXT & & .449 & & & & .478 & & .485 \\
\hline 16 & CEX & & & .725 & & .664 & & .318 & \\
\hline 18 & CEX & & & .683 & & .596 & & .324 & \\
\hline 19 & CIN & .662 & & & .650 & & & .786 & \\
\hline 20 & $\mathrm{CIN}$ & .676 & & & .671 & & & .765 & \\
\hline 21 & CIN & .677 & & & .722 & & & .747 & \\
\hline 22 & CIN & .836 & & & .627 & & & .916 & \\
\hline 23 & $\mathrm{CIN}$ & .699 & & & .571 & & & .771 & \\
\hline 24 & $\mathrm{CIN}$ & .755 & & & .660 & & & .821 & \\
\hline
\end{tabular}

Nota: Método de extracción: Ejes principales. Método de rotación: Promax. Exp. = factor esperado en la configuración factorial: $\mathrm{CEX}=$ Control externo o de la exteriorización de la ira dirigida contra los demás, $\mathrm{CON}=\mathrm{Control}$ interno o de la ira ocultada ante los demás, EXT = Exteriorización o expresión de ira dirigida contra los demás, e $\mathrm{INT}=$ Interiorización o presencia de ira contenida.

\section{Con 24 ítems una vez sustituidos tres} ítems de interiorización por los sugeridos por Moral et al. (2010).

El número de factores fue tres, determinado por el análisis paralelo de Horn, así mismo, el promedio mínimo de las correlaciones parciales al cuadro, tanto en la muestra conjunta como en la muestra de hombres y mujeres. Tras la rotación oblicua se configuró un factor de expresión de la ira con 12 ítems $(2,4,6,7,9,12,13$, $14,15,25,26$ y 27), un factor de control interno con 6 ítems $(19,20,21,22,23$ y 24) y otro de control externo con 6 (ítems 1, 5, 8, 11, 16 y 18) en las tres muestras. 
Contraste del ajuste en la muestra conjunta e invarianza entre ambos sexos de los dos modelos alternativos derivados del análisis factorial exploratorio

Modelo de tres factores correlacionados con 22 ítems.

Se contrastó un modelo de tres factores correlacionales: control interno con seis indicadores, control externo con seis indicadores y expresión de ira con 10 indicadores. En la muestra conjunta (análisis unigrupo), la solución fue admisible. Todos los parámetros fueron significativos por ML y PCS. Las tres correlaciones fueron menores que .70. La bondad de ajuste fue rechazada por la prueba chicuadrada y de Bollen-Stine. Los demás índices mostraron un ajuste aceptable (ver tabla 5).
Al contrastar la invarianza del modelo entre ambos sexos (análisis multigrupo), las soluciones fueron admisibles. Todos los parámetros fueron significativos en los cuatro modelos anidados y las dos muestras por ML y PCS, salvo el peso del factor de expresión sobre el ítem cuatro en el modelo sin constricciones en mujeres por ML y PCS, y en hombres por ML. La correlación entre control interno y control externo fue mayor que .70 en los modelos sin constricciones y con constricciones en los pesos de medida en hombres. Todas las demás correlaciones fueron menores que .70. La bondad de ajuste se rechazó por la prueba chi-cuadrada y de BollenStine, pero los demás índices reflejaron un ajuste aceptable en el modelo sin constricciones. El ajuste empeoró significativamente en los modelos con constricciones (ver tabla 5).

Tabla 5

Índices de ajuste del modelo de tres factores correlacionados (sin items 3 y 17)

\begin{tabular}{cccccc}
\hline Índices & Uni- & \multicolumn{4}{c}{ Multigrupo (mujeres y hombres) } \\
\cline { 3 - 5 } de ajuste & grupo & SC & PM & VE & RM \\
\hline$X^{2}$ & 479.346 & 793.70 & 827.96 & 843.83 & 878.14 \\
$G I$ & 206 & 412 & 431 & 437 & 459 \\
$P$ & $<.001$ & $<.001$ & $<.001$ & $<.001$ & $<.001$ \\
$X^{2} / g l$ & 2.327 & 1.926 & 1.921 & 1.931 & 1.913 \\
$p$ de B-S & 0 & .001 & .001 & .001 & .001 \\
CFI & .841 & .806 & .788 & .782 & .776 \\
RMSEA & .075 & .062 & .062 & .063 & .062 \\
SRMR & .073 & .087 & .092 & .097 & .099 \\
\hline
\end{tabular}

Nota. Modelos anidados con constricciones acumulativas: $\mathrm{SC}=$ sin constricciones, $\mathrm{PM}=$ con constricciones en pesos de medida, $\mathrm{VE}=$ en varianzas-covarianzas estructurales y $\mathrm{RM}=$ en residuos de medida. 
La bondad de ajuste del modelo original y la del modelo de cuatro factores con los tres ítems de interiorización sustituidos, fueron significativamente peores que las del modelo de tres factores con 22 ítems $\left(\Delta \chi^{2}[40, n=240]=88.99, p\right.$ $<.001$ y $\Delta \chi^{2}[40, N=240]=104.63, p<.001$, respectivamente), empero, la bondad de ajuste de dicho modelo de tres factores con 22 ítems fue significativamente en comparación con el modelo de cuatro factores con 22 ítems $\left(\Delta \chi^{2}[3\right.$, $N=240]=27.89, p<.001)$.

Modelo de tres factores con 24 ítems (con tres ítems de interiorización sustituidos).

Al contrastar el modelo de tres factores con 24 ítems (con tres ítems de interiorización sustituidos) en la muestra conjunta (análisis unigrupo), la solución fue admisible. Todos los parámetros fueron significativos por ML y PCS. Las correlaciones fueron menores que .70. La bondad de ajuste se rechazó por la prueba chicuadrada y de Bollen-Stine. Los demás índices mostraron un ajuste aceptable (ver tabla 6). Tuvo una bondad de ajuste significativamente peor que la de los otros cuatro modelos.

Al contrastar la invarianza del modelo entre ambos sexos (análisis multigrupo), las soluciones fueron admisibles. Todos los parámetros fueron significativos en los cuatro modelos $y$ las dos muestras por ML y PCS, a excepción del peso del factor de exteriorización sobre el ítem cuatro en el modelo sin constricciones en mujeres, también, determinado por ambos métodos. Todas las demás correlaciones fueron menores que .70. Los índices de ajuste fueron malos en el modelo sin constricciones y éstos empeoraron en los modelos con constricciones, excepto RMSEA y $\chi 2 / g l$ (ver tabla 6).

Tabla 6

Índices de ajuste del modelo de tres factores correlacionados con tres items de interiorización sustituidos

\begin{tabular}{cccccc}
\hline Índices & Uni- & \multicolumn{4}{c}{ Multigrupo (mujeres y hombres) } \\
\cline { 3 - 6 } de ajuste & grupo & $\mathrm{SC}$ & $\mathrm{PE}$ & $\mathrm{VE}$ & $\mathrm{RE}$ \\
\hline $\mathrm{X}^{2}$ & 627.604 & 997.444 & 1027.075 & 1033.381 & 1086.355 \\
$g l$ & 249 & 498 & 519 & 525 & 549 \\
$p$ & $<.001$ & $<.001$ & $<.001$ & $<.001$ & $<.001$ \\
$X^{2} / g l$ & 2.520 & 2.003 & 1.979 & 1.968 & 1.979 \\
$p$ de B-S & 0 & 0 & 0 & 0 & 0 \\
CFI & .820 & .781 & .777 & .777 & .764 \\
RMSEA & .080 & .065 & .064 & .064 & .064 \\
SRMR & .079 & .093 & .098 & .100 & .104 \\
\hline
\end{tabular}

Nota. Modelos con constricciones anidadas: $\mathrm{SC}=$ sin constricciones, $\mathrm{PM}=$ con constricciones en pesos de medida, $\mathrm{VE}=$ en varianzas-covarianzas estructurales y $\mathrm{RM}=$ en residuos de medida. 


\section{Discusión}

Como en el estudio de Moral et al. (2010), los ítems tres: "me guardo para mí lo que siento" y 17: "evito enfrentar aquello que me enfada", mostraron problemas de consistencia interna. Aunque el ítem 10 "tiendo a tener rencores que no cuento a nadie", no mostró problemas de consistencia interna ni de configuración factorial, resultó un ítem poco consistente en comparación con los tres nuevos ítems introducidos. No obstante, la sustitución de estos ítems 3, 10 y 17 por los nuevos ítems no arrojó ventajas en la configuración, ajuste e invarianza entre ambos sexos del modelo de cuatro factores correlacionados. Por el contrario, la eliminación de los ítems 3 y 17 parece dar el mejor resultado. La mayor inconsistencia de los ítems 3 y 17 frente a los ítems 7 "ardo por dentro aunque no lo demuestro", 12: "estoy más enfadado/a de lo que quiero admitir" y 14: "me irrito más de lo que la gente se piensa", probablemente se deba a su falta de especificidad ante el rasgo evaluado, guardarse u ocultar la ira que se siente ante los demás sin implicar necesariamente huir (estrategia de evitación ante conflictos) o guardarse todos los sentimientos (por posible dificultad para identificar y expresar sentimientos, es decir, por posible alexitimia). Una redacción más específica del rasgo podría mejorar la consistencia interna de estos dos ítems, por ejemplo, "me guardo el enojo que siento para mí", en el caso del ítem tres y "me callo y no digo nada ante aquello que me enfada", para el ítem 17.
La propuesta de cinco factores de Alcázar et al. (2011) no resultó apoyada por los daos expuestos. Debe señalarse que la consistencia interna de los dos factores no esperados de evitación de las otras personas y sentimientos contenidos/rumiación era cuestionable o pobre. La descomposición del factor de interiorización y la configuración de estos dos factores reflejan el problema de consistencia interna que existe dentro de dicho factor. Este problema de consistencia interna sí apareció en este estudio, al igual que en estudios previos (Moral et al., 2010; Moral \& Segovia, 2015; Moscoso \& Spielberger, 1999; Oliva et al., 2010); en el presente estudio, esto se atribuye a los ítems 3 y 17.

Aunque el modelo con mejor ajuste $y$ propiedades de invarianza entre ambos sexos fue el de cuatro factores correlacionados sin los ítems 3 y 17, debe considerarse que la distinción entre interiorización y exteriorización es artificiosa. En el análisis factorial confirmatorio, la correlación entre interiorización y exteriorización fue muy alta; el análisis paralelo de Horn y el promedio mínimo de las correlaciones parciales al cuadrado mostraron que sólo tres factores serían sustantivos. Al igual que en el estudio de Moral y Segovia (2015), se obtuvo que estos tres factores son los dos esperados de control y uno nuevo de expresión de ira, resultado de la unión de los ítems de interiorización y exteriorización. Además, al unir los seis ítems de exteriorización y los cuatro de interiorización ( $\sin$ los dos ítems 
con problemas de consistencia interna, ítems 3 y 17), se logró un factor cuya consistencia interna fue aceptable cuando era pobre.

Los datos reflejan que mujeres y hombres no parecen diferenciar claramente entre la exteriorización y el ocultar la ira que se siente, tal vez porque las personas tienden a expresar parcialmente su ira y guardarse parte de ella para sí mismos, como respuesta a la desaprobación de la conducta irascible (Carlozzi et al., 2010; McEwan, Davis, MacKenzie, \& Mullen, 2009).

Debe señalarse que los valores de ajuste del presente estudio fueron semejantes a los reportados por estudios anteriores, como el de Moral et al. (2010), Moral y Segovia (2015) y Oliva et al. (2010), y deben juzgarse como aceptables desde los índices $\chi^{2} / g l, R M S E A$, SRMR y CFI. Hayduk, Cummings, Boadu, Pazderka-Robinson y Boulianne (2007) señalan que, ante un modelo factorial complejo con un número grande de indicadores, es muy difícil lograr un buen ajuste a los datos, ya que el modelo presenta una parsimonia altísima y es posible perder información. Los índices de ajuste más sensibles a esta complejidad son la prueba chi-cuadrada, afectada a su vez por el incumplimiento de la normalidad multivariada, y la prueba de Bollen-Stine, aunque esta última no requiere del supuesto de normalidad multivariada (Kenny \& McCoach, 2003). Los índices más robustos frente a esta complejidad, y cuyo reporte es mayormente recomendado, son los índices: $\chi^{2} / g l, R M S E A$, SRMR y CFI (Sharma, Mukherjee, Kumar, \&
Dillon, 2005), los cuales muestran un ajuste aceptable.

Como limitación del estudio debe señalarse el empleo de una muestra no probabilística, por lo tanto los resultados deben manejarse como hipótesis para futuros estudios y no como estimaciones de parámetros válidas para adultos de población general mexicana.

En conclusión, los ítems 3 y 17 deben ser eliminados por problemas de consistencia interna, con lo cual se logra una consistencia aceptable en el factor de interiorización al que pertenecen y se incrementa la consistencia interna de la escala, que es buena. No parece traer ventajas la sustitución de los ítems incluidos en el presente estudio. La estructura factorial de la escala reducida a 22 ítems es de tres factores correlacionados: control de la ira manifiesta, control de la ira que se oculta ante los demás y manifestación de la ira (unión de interiorización y exteriorización sin los ítems 3 y 17), tal como lo indican tanto la correlación muy alta entre interiorización y exteriorización en el análisis factorial confirmatorio como el análisis paralelo de Horn y el criterio de Velicer. La consistencia interna de estos tres factores fue buena $o$ aceptable. El ajuste a los datos y las propiedades de invarianza en ambas muestras del modelo de tres factores correlacionados fueron aceptables desde los índices $\chi 2 / g l, R M S E A, S R M R$ y CFI.

Se sugiere modificar la redacción de los ítems 3 y 17 para hacerlos más específicos al rasgo y así probar la configuración sustantiva de cuatro 
factores con mejor ajuste que el modelo de tres factores. Se recomienda en futuros estudios de validación de la escala AX del STAXI-2 reportar la correlación entre estos dos factores de interiorización y exteriorización ante la posibilidad de que sea artificiosa su distinción y aplicar el análisis paralelo de Horn y el criterio de Velicer para definir el número de factores.

\section{Referencias}

Alcázar, R. J., Deffenbacher, J. L., \& Byrne, Z. S. (2011). Assessing the Factor Structure of the Anger Expression Inventory (MLSTAXI) in a Mexican Sample. International Journal of Psychology and Psychological Therapy, 11, 307-318.

Borteyrou, X., Bruchon-Schweitzer, M., \& Spielberger, C. D. (2008) The French adaptation of the STAXI-2, C. D. Spielberger's State-trait anger expression inventory. Encephale, 34, 249-255. doi: 10.1016/j.encep.2007.06.001

Byrne, B. M. (2010). Structural Equation Modeling With AMOS: Basic Concepts, Applications, and Programming. New York: Taylor \& Francis.

Carlozzi, B., Winterowd, C., Harrist, S., Thomason, N., Bratkovich, K., \& Worth, S. (2010). Spirituality, Anger, and Stress in Early Adolescents. Journal of Religion and Health, 49(4), 445-59. doi: 10.1007/s10943-0099295-1
Carver, C. S., \& Harmon-Jones, E. (2009). Anger is an approach-related affect: evidence and implications. Psychology Bulletin, 135, 183-204. doi: 10.1037/a0013965

Cronbach, L. J., \& Shavelson, R. J. (2004). My current thoughts on coefficient alpha and successor procedures. Educational and Psychological Measurement, 64, 391-418. doi: 10.1177/0013164404266386

Del Barrio, V., Aluja, A., \& Spielberger, C. (2004). Anger assessment with the STAXICA: psychometric properties of a new instrument for children and adolescents. Personality and Individual Differences, 37, 227-244. doi:10.1016/j.paid.2003.08.014

Etzler, S. L., Rohrmann, S., \& Brandt, H. (2014). Validation of the STAXI-2: A study with prison inmates. Psychological Test and Assessment Modeling, 56, 178-194.

Hayduk, L., Cummings, G. G., Boadu, K., Pazderka-Robinson, H., \& Boulianne, S. (2007). Testing! Testing! One, two three-Testing the theory in structural equation models! Personality and Individual Differences, 42, 841850. doi: 10.1016/j.paid.2006.10.001

Kenny, D. A., \& McCoach, D. B. (2003). Effect of the number of variables on measures of fit in structural equation modeling. Structural Equation Modeling, 10, 333-351. doi:10.1207/S15328007SEM1003_1

McEwan, T. E., Davis, M. R., MacKenzie, R., \& Mullen, P. E. (2009). The effects of social desirability response bias on STAXI-2 
profiles in a clinical forensic sample. British Journal of Clinical Psychology, 48, 431-436. doi:10.1348/014466509X454886

Miguel Tobal, J. J., Casado, M. I., Cano, A., \& Spielberger, C. D. (2006). STAXI-2: Inventario de Expresión de Ira Estado-Rasgo. Madrid: TEA.

Moral, J., González, M., \& Landero, R. (2010). Factor structure of the STAXI-2-AX and its relationship to the burnout in housewives. The Spanish Journal of Psychology, 13, 418430. doi:10.1017/S1138741600003978

Moral, J., \& Segovia, M. P. (2015). Propiedades psicométricas de la escala de expresión de la ira en mujeres con VIH. Revista Ciencia UANL, 18(74), 69-74.

Moscoso, M. S. (2000). Estructura factorial del Inventario Multicultural Latinoamericano de la Expresión de la Cólera y la Hostilidad. Revista Latinoamericana de Psicología, 32, 321-343.

Moscoso, M. S., \& Spielberger, C. D. (1999). Evaluación de la experiencia, expresión y control de la cólera en Latinoamérica. Psicología Contemporánea, 6(1), 4-21.

Oliva, F. J., Hernández, M. R., \& Calleja, N. (2010). Validación de la versión mexicana del Inventario de Expresión de Ira EstadoRasgo (STAXI-2). Acta Colombiana de Psicología, 13(2), 107-117.

Sharma, S., Mukherjee, S., Kumar, A., \& Dillon, W. R. (2005). A simulation study to investigate the use of cutoff values for assessing model fit in covariance structure models. Journal of Business Research, 58, 935-943. doi: 10.1016/j.jbusres.2003.10.007 Sociedad Mexicana de Psicología. (2007). Código ético del psicólogo ( $4^{a}$ ed.). Ciudad de México: Trillas.

Spielberger, C. D. (1999). The State-Trait Anger Expression Inventory-2: Professional manual. Odessa, FL: Psychological Assessment Resources.

Welty, J. P. (Ed.). (2011). Psychology of anger: Symptoms, causes and coping. New York, NY: Nova Science Publisher.

Recibido el 10 de abril de 2015

Revisado el 27 de abril de 2015 Aceptado el 30 de julio de 2015 


\section{Apéndice \\ Escala de Expresión de Ira (AX)}

A continuación se presenta una serie de afirmaciones que la gente usa para describir sus reacciones cuando se siente enfadada. Lea cada afirmación y rodee con un círculo el número que mejor indique CÓMO REACCIONA O SE COMPORTA CUANDO ESTÁ ENFADADO/A O FURIOSO/A, utilizando la siguiente escala:

\begin{tabular}{|c|c|c|c|c|c|c|c|}
\hline & 1 & 2 & \multicolumn{2}{|l|}{3} & \multicolumn{3}{|c|}{4} \\
\hline & Casi nunca & Algunas veces & \multicolumn{2}{|l|}{ A menudo } & \multicolumn{3}{|c|}{ Casi siempre } \\
\hline 1 & \multicolumn{3}{|c|}{ Controlo mi temperamento. } & 1 & 2 & 3 & 4 \\
\hline 2 & \multicolumn{3}{|c|}{ Expreso mi ira. } & 1 & 2 & 3 & 4 \\
\hline 3 & \multicolumn{3}{|c|}{ Me guardo para mí lo que siento. } & 1 & 2 & 3 & 4 \\
\hline 4 & \multicolumn{3}{|c|}{ Hago comentarios irónicos de los demás. } & 1 & 2 & 3 & 4 \\
\hline 5 & \multicolumn{3}{|c|}{ Mantengo la calma. } & 1 & 2 & 3 & 4 \\
\hline 6 & \multicolumn{3}{|c|}{ Hago cosas como dar portazos. } & 1 & 2 & 3 & 4 \\
\hline 7 & \multicolumn{3}{|c|}{ Ardo por dentro aunque no lo demuestro. } & 1 & 2 & 3 & 4 \\
\hline 8 & \multicolumn{3}{|c|}{ Controlo mi comportamiento. } & 1 & 2 & 3 & 4 \\
\hline 9 & \multicolumn{3}{|c|}{ Discuto con los demás. } & 1 & 2 & 3 & 4 \\
\hline 10 & \multicolumn{3}{|c|}{ Tiendo a tener rencores que no cuento a nadie. } & 1 & 2 & 3 & 4 \\
\hline 11 & \multicolumn{3}{|c|}{ Puedo controlarme y no perder los estribos. } & 1 & 2 & 3 & 4 \\
\hline 12 & \multicolumn{3}{|c|}{ Estoy más enfadado/a de lo que quiero admitir. } & 1 & 2 & 3 & 4 \\
\hline 13 & \multicolumn{3}{|c|}{ Digo barbaridades. } & 1 & 2 & 3 & 4 \\
\hline 14 & \multicolumn{3}{|c|}{ Me irrito más de lo que la gente se cree. } & 1 & 2 & 3 & 4 \\
\hline 15 & \multicolumn{3}{|c|}{ Pierdo la paciencia. } & 1 & 2 & 3 & 4 \\
\hline 16 & \multicolumn{3}{|c|}{ Controlo mis sentimientos de enfado. } & 1 & 2 & 3 & 4 \\
\hline 17 & \multicolumn{3}{|c|}{ Rehúyo encararme con aquello que me enfada. } & 1 & 2 & 3 & 4 \\
\hline 18 & \multicolumn{3}{|c|}{ Controlo el impulso de expresar mis sentimientos de ira. } & 1 & 2 & 3 & 4 \\
\hline 19 & \multicolumn{3}{|c|}{ Respiro profundamente y me relajo. } & 1 & 2 & 3 & 4 \\
\hline 20 & \multicolumn{3}{|c|}{ Hago cosas como contar hasta diez. } & 1 & 2 & 3 & 4 \\
\hline 21 & \multicolumn{3}{|c|}{ Trato de relajarme. } & 1 & 2 & 3 & 4 \\
\hline 22 & \multicolumn{3}{|c|}{ Hago algo sosegado para calmarme. } & 1 & 2 & 3 & 4 \\
\hline 23 & \multicolumn{3}{|c|}{ Intento distraerme para que se me pase el enfado. } & 1 & 2 & 3 & 4 \\
\hline 24 & \multicolumn{3}{|c|}{ Pienso en algo agradable para tranquilizarme. } & 1 & 2 & 3 & 4 \\
\hline 25 & \multicolumn{3}{|c|}{ Me duele cabeza por la rabia que siento. } & 1 & 2 & 3 & 4 \\
\hline 26 & \multicolumn{3}{|c|}{ Me siento cansado/a por el enojo que siento. } & 1 & 2 & 3 & 4 \\
\hline 27 & \multicolumn{3}{|c|}{ Grito por dentro de rabia. } & 1 & 2 & 3 & 4 \\
\hline
\end{tabular}

